\title{
Ultra-thin 2D transition metal monochalcogenide crystals by planarized reactions
}

\author{
Hao-Ting Chin ${ }^{1}$, Mario Hofmann $\mathbb{D}^{2}{ }^{2}$, Su-Yu Huang ${ }^{3}$, Song-Fu Yao ${ }^{4}$, Jian-Jhang Lee ${ }^{2}$, Chia-Chun Chen ${ }^{4}$, Chu-Chi Ting ${ }^{3}$ and \\ Ya-Ping Hsieh iD ${ }^{1,3 凶}$
}

We here present a planarized solid-state chemical reaction that can produce transition metal monochalcogenide (TMMC) 2D crystals with large lateral extent and finely controllable thickness down to individual layers. The enhanced lateral diffusion of a gaseous reactant at the interface between a solid precursor and graphene was found to provide a universal route towards layered TMMCs of different compositions. A unique layer-by-layer growth mechanism yields atomically abrupt crystal interfaces and kinetically controllable thickness down to a single TMMC layer. Our approach stabilizes 2D crystals with commonly unattainable thermodynamic phases, such as $\beta-\mathrm{Cu}_{2} \mathrm{~S}$ and $\gamma$-CuSe, and spectroscopic characterization reveals ultra-large phase transition depression and interesting electronic properties. The presented ability to produce large-scale $2 \mathrm{D}$ crystals with high environmental stability was applied to highly sensitive and fast optoelectronic sensors. Our approach extends the morphological, compositional, and thermodynamic complexity of $2 \mathrm{D}$ materials.

npj 2D Materials and Applications (2021)5:28; https://doi.org/10.1038/s41699-021-00207-2

\section{INTRODUCTION}

The fundamental effect of dimensional confinement on electrons is exemplified in $2 D$ materials, which restrict their wave functions to atomic dimensions. Extending the thickness of a 2D material has been shown to fundamentally alter its properties as evidenced by changes to the band structure ${ }^{1}$, metallicity ${ }^{2}$, thermodynamic phase $^{3}$, and superconducitivity ${ }^{4}$. The gained insight into elementary structure-property relations $s^{5}$ and the prospect of applications in future optoelectronic devices ${ }^{6}$ has attracted significant interest in the realization of $2 \mathrm{D}$ materials multi-layers and van-der-Waals crystals $^{7,8}$. Such structures represent specific examples of a class of nanostructures-2D crystals, which are laterally extended and span the thickness range between atomic-scale $2 \mathrm{D}$ materials and mesoscopic crystals of a few nanometers thickness.

To date, however, no method exists to controllably produce 2D crystals at sufficient scale and quality to support such research. Currently, the most common approach to producing 2D crystals with controllable thickness is the thinning of bulk crystals ${ }^{9}$. However, the required exfoliation step results in small crystallites with uncontrollable thickness and location. Alternatively, conversion of thin films, molecular beam epitaxy, or atomic layer deposition can produce ultrathin 2D materials with controlled thickness but they yield small grain sizes and inhomogeneous morphology ${ }^{10}$. Finally, CVD growth generates high-quality materials at large lateral scale but thickness-dependent growth energetics ${ }^{11}$ yields self-limiting synthesis processes of predominantly single-layers or bilayers with little control over thickness or stacking order ${ }^{12}$.

We here present a reaction process that produces $2 \mathrm{D}$ crystals with controllable thickness, high quality, and unique compositional, electronic, and thermodynamic properties. This advance is achieved by conducting solid-state chemical reactions where one reagent exhibits an enhanced lateral diffusion compared to vertical interdiffusion (Fig. 1a) leading to a 'planarized reaction' that favors two-dimensional growth.

Planarizing a chemical reaction between a gaseous and a solid precursor using a graphene layer was demonstrated to produce layered 2D crystals with atomically sharp interfaces. Moreover, the confinement of the reaction at the graphene interface imparted such $2 \mathrm{D}$ crystals with unexpected compositions and provided a general synthesis method of previously unattainable transition metal mono-chalcogenides (TMMCs). Our approach is found to fundamentally differ from conventional CVD as it permits the control of the $2 \mathrm{D}$ crystal thickness by kinetic parameters, such as growth duration. Optimization of the growth process was demonstrated to achieve synthesis of TMMCs with single- and few-layer thickness and micrometer grain size. Surprisingly, several TMMCs exhibited thermodynamically unfavorable phases, such as $\beta-\mathrm{Cu}_{2} \mathrm{~S}$ and $\mathrm{\gamma}-\mathrm{Cu}_{2} \mathrm{~S}$, with ultra-large phase transition temperature depressions and interesting electronic properties. Finally, our realization of high-performance optoelectronic devices highlights the potential of $2 \mathrm{D}$ crystals for future applications.

\section{RESULT AND DISCUSSION Planarized solid-state chemical reaction}

We carry out solid-state chemical reactions of a solid transition metal with a gaseous chalcogenide at $180^{\circ} \mathrm{C}$ (see Methods section for more details). At these low temperatures interdiffusion between the reactants proceeds at a few nanometers per hour ${ }^{13}$ which indicates the potential of this approach to control the thickness of thus produced materials with atomic resolution. While such approaches have been successful at creating nanoparticles with high precision ${ }^{14}$, the isotropic nature of the materials transport in the solid limit the usefulness towards the synthesis of $2 \mathrm{D}$ materials.

\footnotetext{
${ }^{1}$ Institute of Atomic and Molecular Sciences, Academia Sinica, Taipei 106, Taiwan. ${ }^{2}$ Department of Physics, National Taiwan University, Taipei 106 , Taiwan. ${ }^{3}$ Graduate Institute of Opto-Mechatronics, National Chung Cheng University, Chiayi 62102, Taiwan. ${ }^{4}$ Department of Chemistry, National Taiwan Normal University, Taipei 106, Taiwan.

凶email: yphsieh@gate.sinica.edu.tw
} 

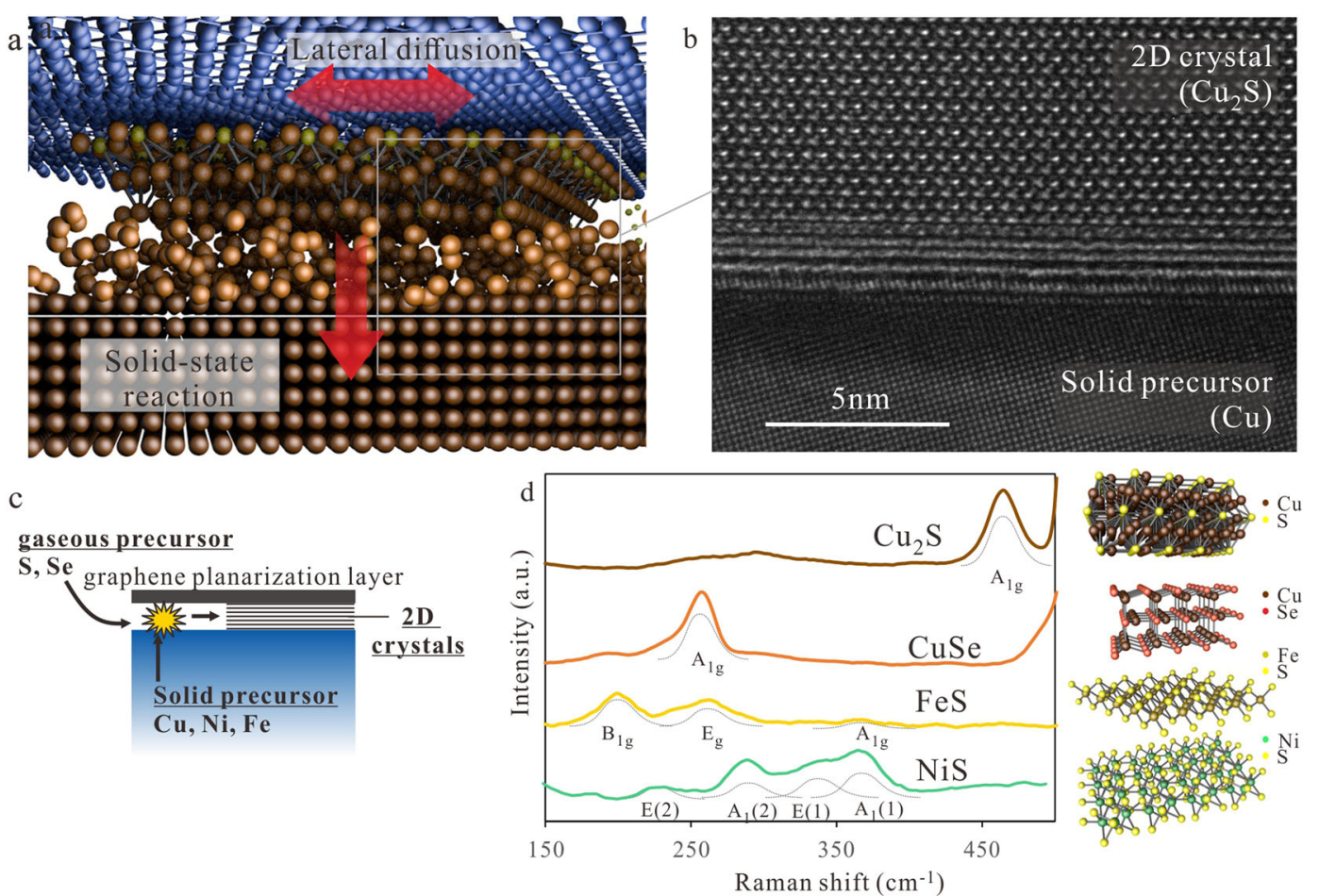

Fig. 1 Planarized solid-state chemical reaction. a Schematic of the planarized reaction process with indications of enhanced lateral diffusion and occuring reaction. $\mathbf{b}$ cross-sectional TEM image of copper/2D crystal interface indicated in $\mathbf{a}$ (the transition region exhibits FIB-induced amorphization of $\mathrm{Cu}^{53}$ ). $\mathbf{c}$ Schematic of experimental realization of 2D crystal growth, d Raman spectra of obtained materials with indication of assigned modes for high-chalcocite $\left(\mathrm{Cu}_{2} \mathrm{~S}\right)^{54}$, covellite $(\mathrm{CuSe})^{55}$, mackinawite $(\mathrm{FeS})^{56}$, millerite (NiS) ${ }^{57}$ and corresponding crystal structure.

To address this issue, we introduce a planarization layer between the solid and gaseous precursors whose attractive vander-Waals force on intercalated gaseous reactants decreases their interdiffusion into the solid compared to the lateral diffusion along its surface (Fig. 1a). Graphene is chosen as the planarization layer, due to its inert structure and strong van-der-Waals interaction $^{15,16}$.

The success of such planarized solid-state chemical reactions can be seen when exposing a graphene-planarized copper crystal to sulfur vapor for $90 \mathrm{~min}$. The produced material has a distinctly different structure than conventional alloys as evidenced by crosssectional transmission electron microscopy (TEM) images (Fig. 1b), which show that the outgrown layer is separated from the substrate by a clear interface. Moreover, the crystal orientation of the outgrown 2D crystal is different from the underlying copper crystal, ruling out the topotactic conversion of the copper.

To demonstrate the universal applicability of the proposed planarized solid-state chemical reaction process, we carry out growth with different transition metal and chalcogen precursors (Fig. 1c). Raman spectroscopic characterization of the resulting materials indicates the formation of layered transition metal mono chalcogenides (TMMCs) in all cases.

These results are surprising, as previous synthesis methods, such as topotactic conversion ${ }^{17,18}$, and epitaxial thin-film deposition $^{19,20}$, could only be applied to produce TMMCs with limited compositions, restricted morphology and insufficient quality for confirming theoretical predictions of TMMC properties ${ }^{21,22}$.

Future work has to identify the reason for the preferential composition, but we hypothesize that this effect may be due to the high van-der-Waals pressure at the graphene/2D crystal interface ${ }^{23}$.

Optical micrographs of the synthesized structures demonstrate large differences in the TMMC morphologies (Supplementary Fig. 2). Whereas copper-based TMMCs, such as CuSe and $\mathrm{Cu}_{2} \mathrm{~S}$, are very thin and laterally extended over large areas, iron- and nickelbased TMMCs, such as FeS and NiS, are relatively thick and exhibit small lateral crystallite sizes. This difference agrees with the low diffusion coefficient of chalcogens in copper compared to other transition metals ${ }^{24}$ and demonstrates the particular suitability of copper for the synthesis of ultrathin 2D crystals, which will be the subsequent focus of this work.

\section{Properties of planarized reactions}

The difference of the planarized-reaction approach from conventional synthesis is further corroborated when comparing the $2 \mathrm{D}$ crystal to material produced without a planarization layer. Conventionally grown $\mathrm{Cu}_{2} \mathrm{~S}$ exhibits $3 \mathrm{D}$ clusters for short growth times that merge into a compact film at long growth times (Fig. 2a and Supplementary Fig. 5). In contrast, planarized growth produces a planar morphology regardless of growth time (Fig. 2b).

The observed independence of $2 \mathrm{D}$ crystal roughness with growth time raises the question about the growth mechanism. Electron absorption provides a simple tool for the visualization of thickness variations in $2 \mathrm{D}$ crystals ${ }^{25}$ and we conduct absorption measurements on freestanding $\mathrm{Cu}_{2} \mathrm{~S}$ crystals that were transferred onto TEM grids by etching of the copper substrate. We observe distinct absorption differences that correspond to individual layers (Fig. 2c) which were found to exhibit fractal boundaries. The observed shapes suggest that each layer is grown independently by a diffusion-limited attachment process $^{26}$. Based on models developed for the initial stages of silicon oxidation $^{27,28}$, we propose that chalcogen atoms, which are intercalated through cracks in the graphene, are freely diffusing at the graphene/copper interface until they attach to a nucleation seed and are then saturated by reaction with copper. Subsequent layers are formed in the same way through reaction of chalcogens that are mobile at the graphene/2D material interface and saturated by copper which diffuses through the grown layer (Fig. 2d). This layer-by-layer growth process through copper outdiffusion can explain the nanometer-scale thickness and may be 

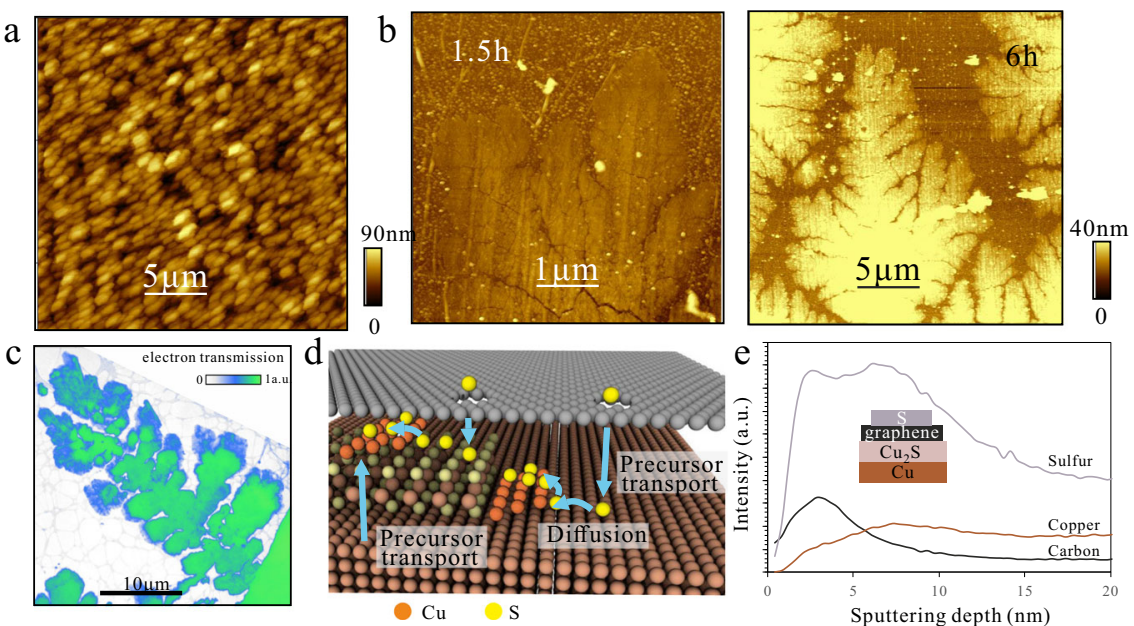

Fig. 2 Properties of planarized reactions. a Atomic force microscopy (AFM) image of copper sulfurized without graphene planarizer, b AFM images of planarized growth results after different growth durations, $\mathbf{c}$ transmission electron absorption micrograph of planarized $\mathrm{Cu}_{2} \mathrm{~S}$. d proposed mechanism of planarized growth. e Secondary lon Mass spectroscopy characterization of material, (inset) schematic of material structure, indicating the presence of elemental sulfur contamination on top of the graphene.
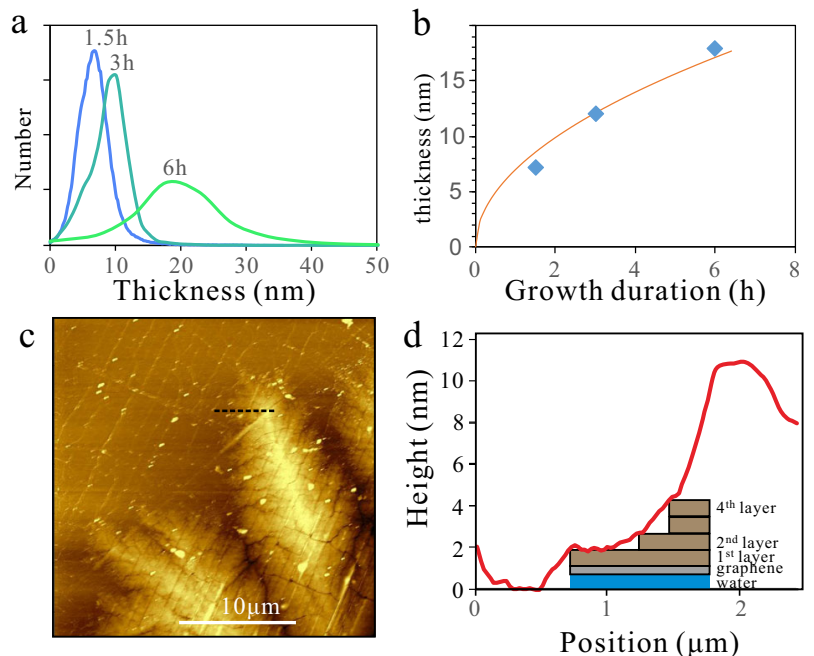

Fig. 3 Thickness control towards single TMMC layers. a Histogram plot of height distribution vs. occurrences for $\mathrm{Cu}_{2} \mathrm{~S}$ crystals obtained after different growth durations, $\mathbf{b}$ fit of time evolution of average thickness to diffusion model described in the text, c AFM image of ultrathin CuSe, $\mathbf{d}$ cross-sectional height distribution indicated in $\mathbf{c}$ and schematic of layer morphology (with graphene and water being drawn as lowest layers for convenience).

assisted by the superionic conduction in $\beta-\mathrm{Cu}_{2} \mathrm{~S}^{29}$ or wrinkles in the graphene $\mathrm{e}^{30}$.

The proposed planarized growth mechanism is furthermore supported by secondary ion mass spectroscopy (SIMS) which shows a lower concentration of copper and a higher concentration of sulfur at the top, indicating the preferential sulfurization from the top (Fig. 2e).

\section{Thickness control towards single TMMC layer}

The presented 2D crystal growth process is fundamentally different from CVD as the thickness of the crystal is controlled by kinetic factors, such as diffusion coefficient, reaction rate, and growth time. Consequently, atomic thickness can be achieved by conducting planarized growth for sufficiently short times. To estimate the achievable lateral 2D crystal size at such thicknesses, we introduce a planarization ratio $(\delta)$ that represents the preference towards lateral growth compared to out-of-plane growth. Assuming a transport limited growth, the planarization can be expressed as

$\delta=\frac{D_{\text {surf }}}{D_{\text {bulk }}}$

where $D_{\text {surf }}$ is the coefficient of surface diffusion and $D_{\text {bulk }}$ is the diffusion coefficient into the bulk. Comparison of surface and bulk diffusion coefficients of sulfur and copper $^{31}$ suggest that planarization ratios of $10^{7}$ could be obtained, indicating the theoretical ability to synthesize single-atomic layers over several centimeters using through such solid-state chemical reactions.

To support these predictions, we carry out planarized growth at varying durations and observe a clear dependence of average sample thickness on growth duration (Fig. 3a). The average thickness $(<t>)$ can be fitted to a simple diffusion model $\langle t\rangle=$ $\sqrt{2 D t}$ (Fig. 3b) and yields a diffusion coefficient (D) of $\mathrm{D}=1.3 \times$ $10^{-16} \mathrm{~cm}^{2} \mathrm{~s}^{-1}$. This parameter is at least four orders of magnitude smaller than the diffusion coefficient of sulfur in copper at the investigated temperatures ${ }^{13}$ which indicates the suppression of interdiffusion by the planarization layer and suggests that even larger values of $\delta$ can be achieved that predicted from bulk diffusion parameters.

Due to its larger atomic radius, we expect that selenium may diffuse even slower than sulfur and investigate the minimum achievable thickness of CuSe 2D crystals. For growth durations of $15 \mathrm{~min}$, CuSe crystals with a significantly lower thickness than $\mathrm{Cu}_{2} \mathrm{~S}$ could be synthesized. Atomic force micrographs (AFM) of CuSe after transfer to Silicon oxide substrates (Fig. 3c) demonstrate a minimum thickness of $1.8 \mathrm{~nm}$ (Fig. 3d). The apparent wrinkles in the AFM image serve as a reminder that the graphene planarization layer is still present and has to be considered when extrapolating towards the thickness of the underlying 2D crystal. Given the out-of-plane spacing between graphite layers $(0.34 \mathrm{~nm})$ and CuSe layers $(0.8 \mathrm{~nm})^{32}$ this thickness is smaller than expected for a stack of graphene/CuSe bilayer and thus represents a stack of single-layer graphene/single-layer CuSe in the presence of surface adsorbates, such as water (inset of Fig. $3 d$ ) $^{33}$.

Our results not only demonstrate the growth of an atomically thin TMMC layer ${ }^{34}$ but the micrometer-scale lateral size of singlelayer regions indicates that the interdiffusion reaction occurs with atomic abruptness over large areas. Moreover, electron diffraction 
a

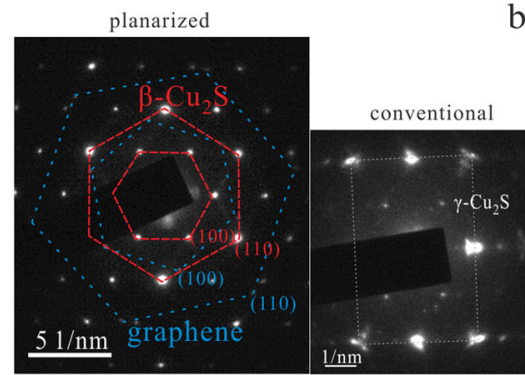

d

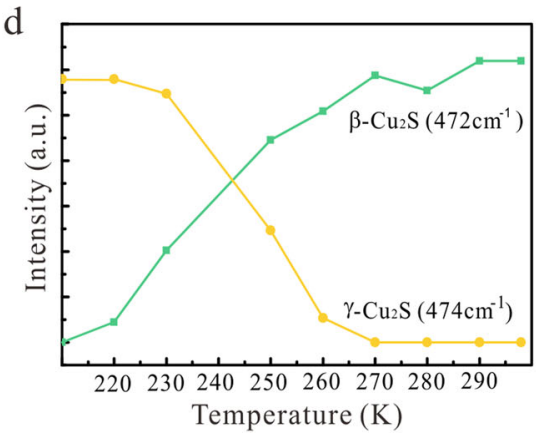

$\mathrm{b}$

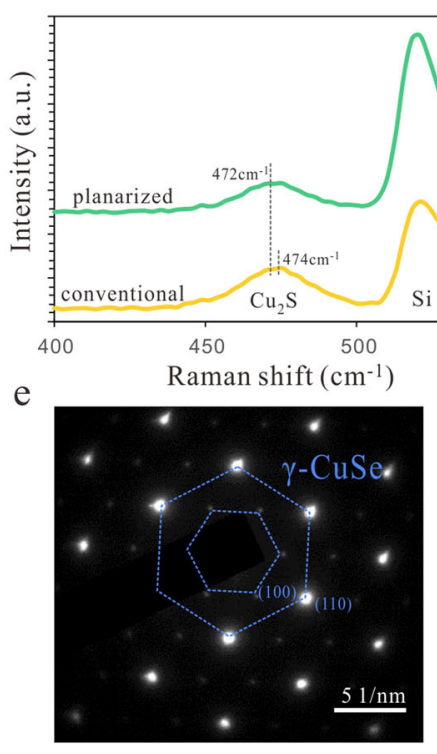

$\mathrm{c}$

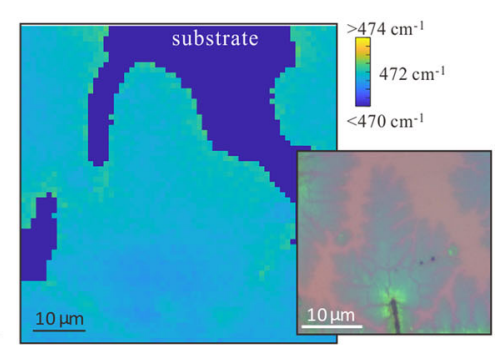
550
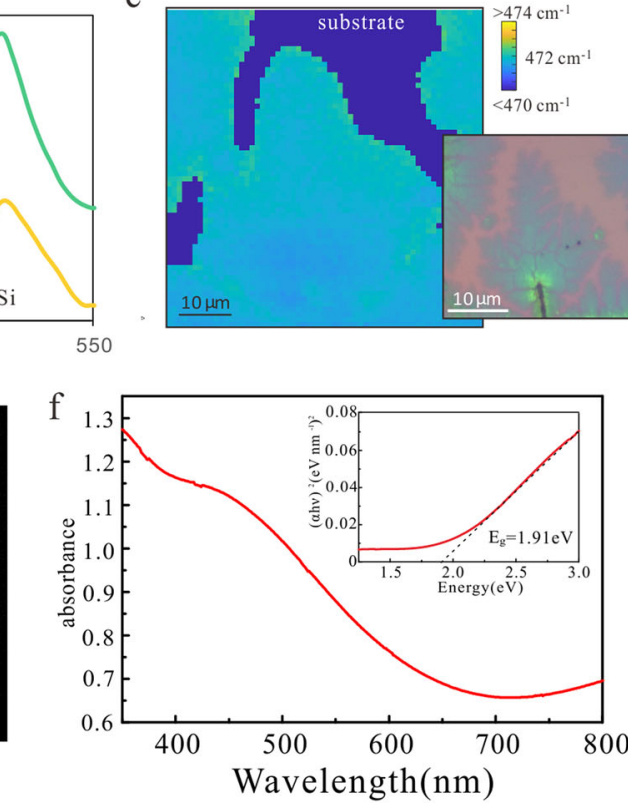

Fig. 4 Features of 2D crystals grown by planarized reaction. a Selected area diffraction (SAED) of $\mathrm{Cu}_{2} \mathrm{~S}$ grown under planarized growth conditions and graphene-free conditions, b Raman spectra of planarized and conventionally grown $\mathrm{Cu}_{2} \mathrm{~S}$, $\mathbf{c}$ Raman $\mathrm{A}_{1 \mathrm{~g}}$ peak position map (the substrate peak position was set to zero) (inset) optical micrograph of $\mathrm{Cu}_{2} \mathrm{~S}$ on $\mathrm{Si} / 90 \mathrm{~nm} \mathrm{SiO} \mathrm{O}_{2}$ d temperature-dependent Raman peak position indicating large phase-transition lowering, e SAED of CuSe, $\mathbf{f}$ absorption measurement, (inset) Tauc Plot with indication of band-gap.

analysis indicates that single-crystal regions extend over several micrometers (Supplementary Fig. 4) which is comparable to the crystallite size of CVD-grown 2D materials, and highlights the potential of applying 2D crystal growth towards large-scale 2D materials synthesis.

\section{Properties of 2D crystals grown by planarized reaction}

In addition to their composition, TMMCs produced by planarized growth exhibit a number of surprising properties. First, selected area electron diffraction (SAED) characterization of freestanding $\mathrm{Cu}_{2} \mathrm{~S}$ reveals two hexagonal lattices (Fig. 4a). The fainter diffraction points correspond to the graphene planarizing layer (the low intensity originating from graphene's atomic thickness). The second pattern belongs to the grown crystal and shows no epitaxial ordering with the graphene, suggesting the growth process to rely on van-der-Waals heteroepitaxy ${ }^{35}$. Moreover, the uniform distribution and intensity of the second diffraction pattern indicates precise stacking between the lattice planes which has been a challenge for conventional 2D materials synthesis $^{36}$.

The extracted lattice parameters for the grown 2D crystal fit within $0.1 \%$ of expected values for $\beta-\mathrm{Cu}_{2} \mathrm{~S}^{37}$. This phase represents a layered arrangement which has not been previously obtained in bulk form at room temperature ${ }^{22}$. Moreover, $\beta-\mathrm{Cu}_{2} \mathrm{~S}$ has been linked to interesting topological ordering ${ }^{38}$ but should not be thermodynamically stable at room temperature. Indeed, conventional growth, produces the thermodynamically stable cubic $\gamma$ phase as confirmed by diffraction measurements (Fig. 4a) and Raman spectroscopy (Fig. 4b).

Under normal growth conditions the synthesized $\beta-\mathrm{Cu}_{2} \mathrm{~S}$ is only stable at high temperatures $(380-700 \mathrm{~K})^{39}$ and could previously only be synthesized by non-equilibrium processing, such as quenching ${ }^{40}$ and surface stabilization ${ }^{20}$. The occurrence of these two mechanisms can be ruled out for planarized growth reactions, based on Raman characterization of 2D crystal regions with different thicknesses (Fig. 4c). We observe a constant peak position, indicative of $\beta-\mathrm{Cu}_{2} \mathrm{~S}$, for both thin and thick regions, which suggests that interfacial strain buildup as observed for surface-stabilized growth $^{41}$ and out-of-equilibrium formation mechanisms ${ }^{40}$ does not occur.

To further investigate the phase transition, we conduct temperature-dependent Raman spectroscopy by extracting the Raman peak intensities of two Lorentzian peaks centered at $472 \mathrm{~cm}^{-1}$ and $474 \mathrm{~cm}^{-1}$, respectively (Fig. 4 d). We observe that at room temperature $\beta-\mathrm{Cu}_{2} \mathrm{~S}$ is formed exclusively and the transition from $\beta$ to $\gamma$ phase $\mathrm{Cu}_{2} \mathrm{~S}$ occurs only at $240 \mathrm{~K}$ which represents a lowering of the phase transition temperature by $140 \mathrm{~K}$ (Fig. 4C).

The large impact of the planarization process on fundamental thermodynamic characteristics is furthermore evidenced when characterizing the SAED of CuSe which indicates the formation of hexagonal gamma-phase $\mathrm{CuSe}^{42}$ (Fig. 4e). Layered, hexagonal CuSe is also not expected to be thermodynamically stable at room temperature and has, to our knowledge, not been synthesized before $^{43}$. The successful realization of hexagonal CuSe can shed light on some of the theoretical predictions on this material, which is considered promising for applications in solar cells ${ }^{44}$, super conductors $^{45}$, and phase change memory ${ }^{46}$. Recent first-principles calculations for covellite-type CuSe ${ }^{47}$ suggested a semimetallic band structure with a $2.6 \mathrm{eV}$ gap between $\mathrm{Cu} 3 \mathrm{~d}$ and S 3p orbitals. Absorption measurements confirm that CuSe exhibits an enhanced interaction for short-wavelength light (Fig. 4f) that indicates an energy gap of $1.9 \mathrm{eV}$.

\section{Application of TMMCs to optoelectronic devices}

Finally, we investigate the potential of applying 2D crystals in optoelectronic devices. The graphene planarization layer was found to not only enable the presented growth approach but imparts the $2 \mathrm{D}$ crystal with impressive environmental stability. Upon exposure of a $\mathrm{Cu}_{2} \mathrm{~S}$ crystal to UV-generated ozone radicals, the graphene is found to significantly deteriorate as evidenced by an increasing Raman D-Band intensity and a decreasing G-Band intensity (Fig. 5a). Even after 150 min exposure, however, the $\mathrm{Cu}_{2} \mathrm{~S}$ $E_{2} g$ peak remains unchanged in position and intensity and no evidence of copper oxide is observed ${ }^{48}$. This chemical robustness represents an advantage of our approach over conventional 

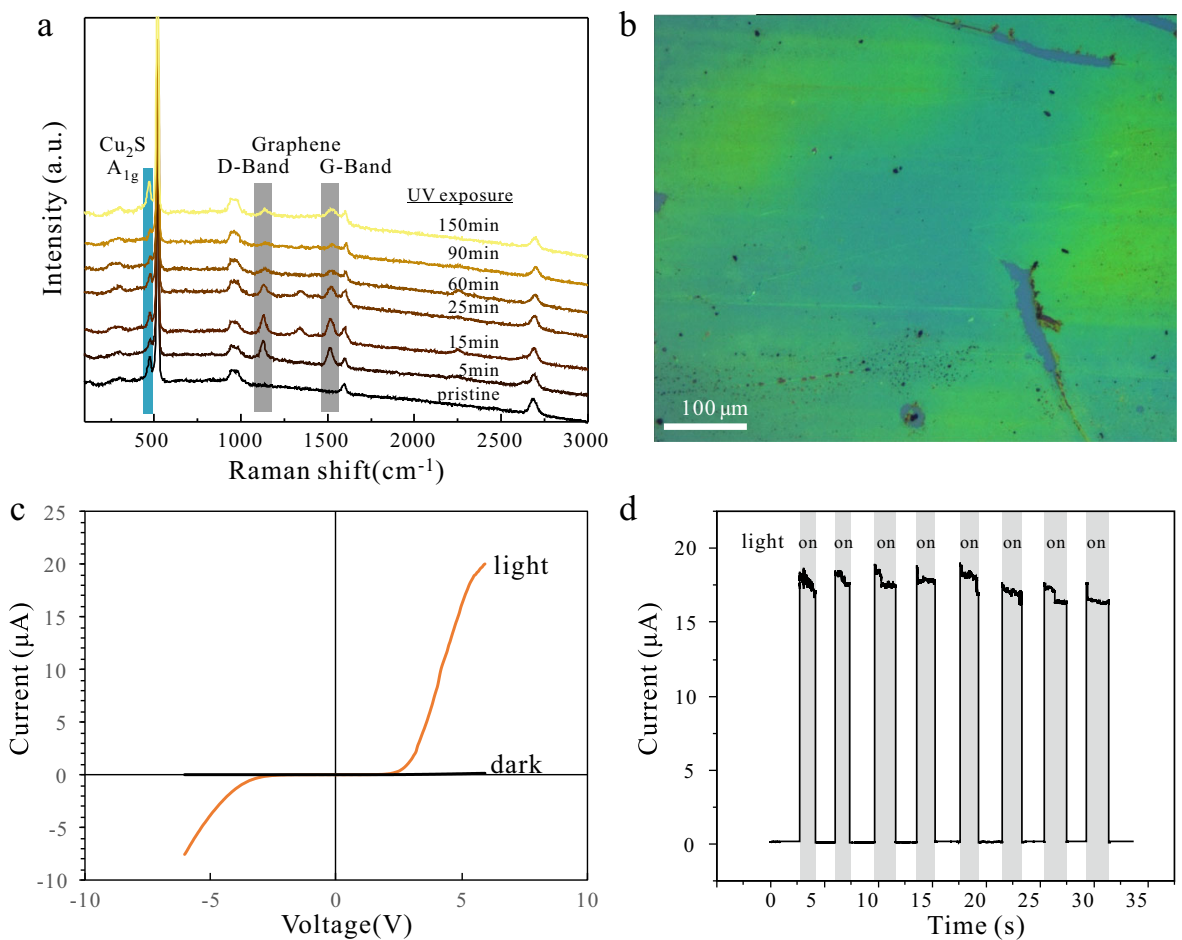

Fig. 5 Application of TMMCs to optoelectronic devices. a Raman spectra after varying duration of ozone exposure, indicating high environmental stability. b Optical micrograph of continuous $\mathrm{Cu}_{2} \mathrm{~S}$ films (tears in the film expose the substrate and are due to the transfer process), c current-voltage characteristics for continuous $\mathrm{Cu}_{2} \mathrm{~S}$ film under dark and illuminated conditions, $\mathbf{d}$ time evolution of photocurrent under pulsed illumination.

TMDCs, which experience significant oxidation at ambient conditions ${ }^{49}$.

While we have so far focused on growth conditions that produce incompletely grown TMMCs, an increase of the growth duration yields continuous and uniform films over large scales (Fig. 5b) which permits the fabrication of optoelectronic devices.

We demonstrate the application of $\mathrm{Cu}_{2} \mathrm{~S}$ to photosensing by comparing the current-voltage characteristics under dark and illuminated conditions (Fig. 5c). The large increase in device current upon illumination and the non-linear IV curve suggest that the sensor's performance is dominated by the response from the semiconducting $\mathrm{Cu}_{2} \mathrm{~S}$ while leakage through the metallic graphene is minimal. Moreover, the detectivity of the device was estimated to be $D^{*}=10^{10}$ Jones which is comparable to state-ofthe-art 2D material photodetectors ${ }^{50}$. Finally, a high carrier mobility can be inferred from the fast response times $(2.7 \mathrm{~ms})$ of the sensors under pulsed illumination (Fig. 5d) which further imparts the sensor with attractive device features.

In conclusion, we have introduced a growth method to produce 2D materials crystals. By conducting a solid-state chemical reaction process at the interface between graphene and a bulk crystal, a planarized reaction is achieved that favors lateral growth over vertical growth with a ratio in excess of 1000. Different from conventional CVD synthesis, the thickness of produced materials is determined by the growth duration and can be adjusted down to a single $2 \mathrm{D}$ material layer. The resulting material exhibits attractive properties that set it apart from MBE and CVD-grown materials, such as atomically precise interfaces, large crystalline domain size, and thickness-independent morphology. Distinctive compositions and unexpected thermodynamic phases highlight the potential of the presented growth process for extending the complexity and potential of 2D materials. Future work will need to establish the applicability to other crystalline substrates and precursors, as well as other 2D materials-based planarization layers. Finally, our approach could be employed in the future to produce complex
2D-material morphologies by modifying the gaseous precursor during growth, for the scalable production of complex van-derWaals heterojunctions.

\section{METHODS}

\section{Synthesis and fabrication}

Monolayer graphene was synthesized on copper foils(Alfa Aesar 46365, purity $99.8 \%$, thickness $25 \mu \mathrm{m})$ by LPCVD using $\mathrm{CH} 4(10 \mathrm{sccm}), \mathrm{H} 2$ (200 sccm) carrier gasses at $1020^{\circ} \mathrm{C}$ following previous reports ${ }^{51}$. Planarized growth was conducted in a 1" quarz tube using a solid chalcogen source (Sulfur, Selenium powder Alfa Aesar, used as received) (Supplementary Fig. 1). For this purpose, both the copper/graphene sample were positioned inside the single heating zone of clamshell furnace. After displacing air with argon flow for $20 \mathrm{~min}$, the chamber was heated to $180^{\circ} \mathrm{C}$ and remained at that temperature for the growth duration.

\section{Characterization}

Transfer of the grown 2D crystals to silicon substrates and TEM grids followed established procedures for graphene transfer ${ }^{52}$, using an ammonium-persulfate-based etchant. Raman spectroscopy was carried out in a home-built confocal micro-Raman system at an excitation wavelength of $532 \mathrm{~nm}$. Transmission Electron microscopy was conducted in Spherical Aberration Corrected Scanning Transmission Electron Microscope. Atomic force microscopy was conducted using a Force AFM Genie E7 and optical microscopy employed an Olympus BX53. Transmittance of 2D crystals was measured on samples that were transferred onto quartz substrates using a Jasco (V-670) UV/visible spectrophotometer.

\section{DATA AVAILABILITY}

The data of this study are available from the corresponding author upon reasonable request. 
Received: 9 July 2020; Accepted: 27 January 2021;

Published online: 05 March 2021

\section{REFERENCES}

1. Ellis, J. K., Lucero, M. J. \& Scuseria, G. E. The indirect to direct band gap transition in multilayered MoS2 as predicted by screened hybrid density functional theory. Appl. Phys. Lett. 99, 261908 (2011).

2. Ciarrocchi, A., Avsar, A., Ovchinnikov, D. \& Kis, A. Thickness-modulated metal-tosemiconductor transformation in a transition metal dichalcogenide. Nat. Commun. 9, 919-919 (2018).

3. Xue, Y. et al. Thickness-dependent magnetotransport properties in $1 \mathrm{~T} \mathrm{VSe2} \mathrm{single}$ crystals prepared by chemical vapor deposition. Nanotechnology 31, 145712 (2020).

4. Wang, H. et al. High-quality monolayer superconductor NbSe 2 grown by chemical vapour deposition. Nat. Commun. 8, 1-8 (2017).

5. Mouri, S. et al. Thermal dissociation of inter-layer excitons in MoS2/MoSe2 heterobilayers. Nanoscale 9, 6674-6679 (2017).

6. Naveh, D. et al. in APS March Meeting Abstracts Vol. 2018 H11.002 (2018).

7. Shi, Z. Y. et al. Vapor-liquid-solid growth of large-area multilayer hexagonal boron nitride on dielectric substrates. Nat. Commun. 11, 2020 (2020).

8. Geim, A. K. \& Grigorieva, I. V. Van der Waals heterostructures. Nature 499, 419-425 (2013).

9. Stanford, M. G., Rack, P. D. \& Jariwala, D. Emerging nanofabrication and quantum confinement techniques for 2D materials beyond graphene. NPJ 2D Mater. Appl. 2, 20 (2018).

10. Heyne, M. H. et al. Multilayer MoS2 growth by metal and metal oxide sulfurization. J. Mater. Chem. C. 4, 1295-1304 (2016).

11. Wang, X. et al. Thickness-controlled synthesis of $\operatorname{CoX} 2(X=S$, Se, and Te) single crystalline $2 \mathrm{D}$ layers with linear magnetoresistance and high conductivity. Chem. Mater. 32, 2321-2329 (2020).

12. Yan, A. et al. Direct growth of single- and few-layer MoS2 on h-BN with preferred relative rotation angles. Nano Lett. 15, 6324-6331 (2015).

13. Fueki, K. \& Ouchi, Y. Solubility and diffusion-coefficient of sulfur in copper. Bull. Chem. Soc. Jpn. 51, 2234-2236 (1978).

14. Shen, N. et al. Solid-state-reaction synthesis of VO2 nanoparticles with low phase transition temperature, enhanced chemical stability and excellent thermochromic properties. Rsc Adv. 5, 108015-108022 (2015).

15. Dienwiebel, M. et al. Superlubricity of graphite. Phys. Rev. Lett. 92, 126101 (2004).

16. Persson, K., Hinuma, Y., Meng, Y. S., Van der Ven, A. \& Ceder, G. Thermodynamic and kinetic properties of the Li-graphite system from first-principles calculations. Phys. Rev. B 82, 125416 (2010).

17. Pan, Q. et al. Reduced graphene oxide-induced recrystallization of NiS nanorods to nanosheets and the improved na-storage properties. Inorg. Chem. 53, 3511-3518 (2014).

18. Gao, T. \& Wang, T. Two-dimensional single crystal CdS nanosheets: synthesis and properties. Cryst. Growth Des. 10, 4995-5000 (2010).

19. Shahzad, R., Kim, T., Mun, J. \& Kang, S. W. Observation of photoluminescence from large-scale layer-controlled $2 \mathrm{D}$ beta-Cu2S synthesized by the vapor-phase sulfurization of copper thin films. Nanotechnology 28, 505601 (2017).

20. Romdhane, F. B. et al. Quasi-2D Cu2S crystals on graphene: in-situ growth and abinitio calculations. Small 11, 1253-1257 (2015).

21. Pandey, D. \& Chakrabarti, A. Prediction of two-dimensional monochalcogenides: MoS and WS. Phys. Lett. A 383, 2914-2921 (2019).

22. Gao, L. et al. Epitaxial growth of honeycomb monolayer CuSe with Dirac nodal line fermions. Adv. Mater. 30, e1707055 (2018).

23. Vasu, K. S. et al. Van der Waals pressure and its effect on trapped interlayer molecules. Nat. Commun. 7, 12168 (2016).

24. Neumann, G. \& Tuijn, C. Self-diffusion and impurity diffusion in pure metals: handbook of experimental data. (Pergamon, 2011).

25. Rubino, S., Akhtar, S. \& Leifer, K. A simple transmission electron microscopy method for fast thickness characterization of suspended graphene and graphite flakes. Microsc. Microanal. 22, 250-256 (2016).

26. Tumbek, L. \& Winkler, A. Attachment limited versus diffusion limited nucleation of organic molecules: Hexaphenyl on sputter-modified mica. Surf. Sci. 606, L55-L58 (2012).

27. Green, M., Gusev, E., Degraeve, R. \& Garfunkel, E. Ultrathin $(<4 \mathrm{~nm}) \mathrm{SiO} 2$ and $\mathrm{Si}-\mathrm{O}-\mathrm{N}$ gate dielectric layers for silicon microelectronics: understanding the processing, structure, and physical and electrical limits. J. Appl. Phys. 90, 2057-2121 (2001).

28. Watanabe, T., Tatsumura, K. \& Ohdomari, I. SiO2/Si interface structure and its formation studied by large-scale molecular dynamics simulation. Appl. Surf. Sci. 237, 125-133 (2004).
29. Balapanov, M. K., Zinnurov, I., Mukhamed'yanov, U. K. \& Musalimov, R. S. Ionic conduction and chemical diffusion in $\mathrm{Cu} 2-\mathrm{x} \mathrm{S}$ codoped with magnesium and aluminum. Inorg. Mater. 43, 449-451 (2007).

30. Vlaic, S. et al. Graphene as a mechanically active, deformable two-dimensional surfactant. J. Phys. Chem. Lett. 9, 2523-2531 (2018).

31. Hinch, B. J., Frenken, J. W. M., Zhang, G. \& Toennies, J. P. Sulfur adatom diffusion on the $\mathrm{Cu}(111)$ surface. Surf. Sci. 259, 288-300 (1991).

32. Berry, L. G. The crystal structure of covellite, cuse and klockmannite, cuse. Am. Mineralogist 39, 504-509 (1954).

33. Shearer, C. J., Slattery, A. D., Stapleton, A. J., Shapter, J. G. \& Gibson, C. T. Accurate thickness measurement of graphene. Nanotechnology 27, 125704 (2016).

34. Wang, H. et al. Integrated circuits based on bilayer MoS2 transistors. Nano Lett. 12, 4674-4680 (2012).

35. Choi, J. E., Yoo, J., Lee, D., Hong, Y. J. \& Fukui, T. Crystal-phase intergradation in InAs nanostructures grown by van der Waals heteroepitaxy on graphene. Appl. Phys. Lett. 112, 142101 (2018).

36. Li, M.-Y., Chen, C.-H., Shi, Y. \& Li, L.-J. Heterostructures based on two-dimensional layered materials and their potential applications. Mater. Today 19, 322-335 (2016).

37. Saitoh, K.-i, Sato, T., Takuma, M., Takahashi, Y. \& Chin, R. Molecular dynamics study on lubrication mechanism in crystalline structure between copper and sulfur. $J$. Mater. 2015, 963257 (2015).

38. Xue, Y. et al. Novel Chern insulators with half-metallic edge states. NPG Asia Mater. 10, e467-e467 (2018).

39. Coughlan, C. et al. Compound copper chalcogenide nanocrystals. Chem. Rev. 117, 5865-6109 (2017).

40. Li, B. et al. Large-size 2D $\beta$-Cu2S nanosheets with giant phase transition temperature lowering $(120 \mathrm{~K})$ synthesized by a novel method of super-cooling chemical-vapor-deposition. Adv. Mater. 28, 8271-8276 (2016).

41. Alaskar, Y., Arafin, S., Martinez-Velis, I. \& Wang, K. L. Heteroepitaxial growth of III-V semiconductors on 2D materials. Two-dimensional Materials-Synthesis, Characterization and Potential Applications (2016).

42. Milman, V. Klockmannite, CuSe: structure, properties and phase stability from ab initio modeling. Acta Crystallogr. Sect. B 58, 437-447 (2002).

43. Glazov, V. M., Pashinkin, A. S. \& Fedorov, V. A. Phase equilibria in the $\mathrm{Cu}$-Se system. Inorg. Mater. 36, 641-652 (2000).

44. Wu, X.-J. et al. Two-dimensional CuSe nanosheets with microscale lateral size: synthesis and template-assisted phase transformation. Angew. Chem. Int. Ed. 53, 5083-5087 (2014).

45. Raveau, B. \& Sarkar, T. Superconducting-like behaviour of the layered Chalcogenides CuS and CuSe below 40 K. Solid State Sci. 13, 1874-1878 (2011).

46. Rehman, S., Kim, K., Hur, J.-H. \& Kim, D.-k Phase transformation induced resistive switching behavior in Al/Cu2Se/Pt. J. Phys. D: Appl. Phys. 50, 135301 (2017).

47. Morales-Garcia, A., Soares, A. L., Dos Santos, E. C., de Abreu, H. A. \& Duarte, H. A. First-principles calculations and electron density topological analysis of covellite (CuS). J. Phys. Chem. A 118, 5823-5831 (2014).

48. Wang, S. H., Huang, Q. J., Wen, X. G., Li, X. Y. \& Yang, S. H. Thermal oxidation of CU2S nanowires: a template method for the fabrication of mesoscopic CuxO $(\mathrm{x}=1,2)$ wires. Phys. Chem. Chem. Phys. 4, 3425-3429 (2002).

49. Li, Q., Zhou, Q. H., Shi, L., Chen, Q. \& Wang, J. L. Recent advances in oxidation and degradation mechanisms of ultrathin $2 \mathrm{D}$ materials under ambient conditions and their passivation strategies. J. Mater. Chem. A 7, 4291-4312 (2019).

50. Mu, C. P., Xiang, J. Y. \& Liu, Z. Y. Photodetectors based on sensitized twodimensional transition metal dichalcogenides: a review. J. Mater. Res. 32, 4115-4131 (2017).

51. Hsieh, Y.-P., Shih, C.-H., Chiu, Y.-J. \& Hofmann, M. High-throughput graphene synthesis in gapless stacks. Chem. Mater. 28, 40-43 (2016).

52. Bhaviripudi, S., Jia, X., Dresselhaus, M. S. \& Kong, J. Role of kinetic factors in chemical vapor deposition synthesis of uniform large area graphene using copper catalyst. Nano Lett. 10, 4128-4133 (2010).

53. Kiener, D., Motz, C., Rester, M., Jenko, M. \& Dehm, G. FIB damage of $\mathrm{Cu}$ and possible consequences for miniaturized mechanical tests. Mater. Sci. Eng. a-Struct. Mater. Prop. Microstruct. Process. 459, 262-272 (2007).

54. Safrani, T., Jopp, J. \& Golan, Y. A comparative study of the structure and optical properties of copper sulfide thin films chemically deposited on various substrates. Rsc Adv. 3, 23066-23074 (2013).

55. Xue, C. et al. Micro-Raman study of orientation effects of CuxSe-crystallites on Curich CuGaSe2 thin films. J. Appl. Phys. 96, 1963-1966 (2004).

56. Genchev, G. \& Erbe, A. Raman spectroscopy of mackinawite FeS in anodic iron sulfide corrosion products. J. Electrochem. Soc. 163, C333-C338 (2016).

57. Wang, J. H., Cheng, Z., Bredas, J. L. \& Liu, M. L. Electronic and vibrational properties of nickel sulfides from first principles. J. Chem. Phys. 127, 214705 (2007). 


\section{ACKNOWLEDGEMENTS}

Y.H. acknowledges funding from Academia Sinica (AS-iMATE-108-32) and the Ministry of Science and Technology.

\section{AUTHOR CONTRIBUTIONS}

Y. H. proposed the idea and supervised the project, H.C. analyzed data, S.H. conduct the experiments, C.T. contributed experimental resources and critical review, M.H. aided in analysis and visualization.

\section{COMPETING INTERESTS}

The authors declare no competing interests.

\section{ADDITIONAL INFORMATION}

Supplementary information The online version contains supplementary material available at https://doi.org/10.1038/s41699-021-00207-2.

Correspondence and requests for materials should be addressed to Y.-P.H.
Reprints and permission information is available at http://www.nature.com/ reprints

Publisher's note Springer Nature remains neutral with regard to jurisdictional claims in published maps and institutional affiliations.

(C) Open Access This article is licensed under a Creative Commons Attribution 4.0 International License, which permits use, sharing, adaptation, distribution and reproduction in any medium or format, as long as you give appropriate credit to the original author(s) and the source, provide a link to the Creative Commons license, and indicate if changes were made. The images or other third party material in this article are included in the article's Creative Commons license, unless indicated otherwise in a credit line to the material. If material is not included in the article's Creative Commons license and your intended use is not permitted by statutory regulation or exceeds the permitted use, you will need to obtain permission directly from the copyright holder. To view a copy of this license, visit http://creativecommons.org/licenses/by/4.0/.

(c) The Author(s) 2021 